\title{
The incidence and outcomes of patients with acute kidney injury in a multidisciplinary intensive care unit in Durban, South Africa
}

\author{
M A Khuweldi, ${ }^{1}$ MB ChB, FCP (SA), MMed (Int Med); OrcID 0000-0002-7295-7317; \\ D L Skinner, ${ }^{2}$ MB ChB, FCS (SA), MMed (Surg), Cert Critical Care (SA); OrcID 0000-0002-2769-2436; \\ K de Vasconcellos, ${ }^{3} \mathrm{MB}$ ChB, FCA (SA), Cert Critical Care (SA); OrcID 0000-0002-1974-6633
}

\begin{abstract}
${ }^{1}$ Department of Internal Medicine, Nelson R Mandela School of Medicine, School of Clinical Medicine, University of KwaZulu-Natal, Durban, South Africa ${ }^{2}$ Department of Critical Care, King Edward VIII Hospital; Discipline of Surgery and Critical Care, School of Clinical Medicine, University of KwaZuluNatal, Durban, South Africa

${ }^{3}$ Department of Critical Care, King Edward VIII Hospital and Discipline of Anaesthesiology and Critical Care, School of Clinical Medicine, University of KwaZulu-Natal, Durban, South Africa
\end{abstract}

Corresponding author: M A Khuweldi (mohamed.khuweldi@gmail.com)

\begin{abstract}
Background. Acute kidney injury (AKI) in critically ill and resource-limited settings is under investigated.
Objectives. To describe the incidence, outcomes and healthcare burden of AKI in a multidisciplinary intensive care unit (ICU) in Durban, South Africa (SA).

Methods. All adult patients admitted to the ICU at King Edward VIII Hospital from January 2016 to June 2016, who did not have end-stage renal disease and survived for more than 6 hours after admission were evaluated for AKI using the kidney disease improving global outcomes (KDIGO) creatinine criteria. Potential risk factors for AKI and an association between AKI and outcomes including ICU mortality and length of stay were analysed.

Results. We screened 204 patients for inclusion into the study and 26 patients were excluded. About half of the patients (50.5\%; $n=90 / 178)$ who were included in the study were diagnosed with AKI at the time of admission and $16.3 \%$ ( $n=29 / 178$ developed AKI in the ICU. Among the patients who had AKI on admission, 50\% ( $n=45 / 90)$ were classified as KDIGO stage1, 21.1\% $(n=19 / 90)$ as stage 2 and $28.8 \%(n=26 / 90)$ as stage 3 . Less than one-third $(24.7 \% ; n=44 / 178)$ of the patients who developed AKI in the ICU were classified as KDIGO stage $1,14 \%(n=25 / 178)$ were stage 2 , and $28 \%(n=50 / 178)$ were stage 3 . The mortality rate for patients with AKI on admission was $40.0 \%(n=36 / 90)$ compared with $39.8 \%$ ( $n=35 / 88)$ for those without AKI on admission ( $p=0.975)$. The mortality rate for all patients with AKI was $46.2 \%(n=55 / 119)$ compared with $27.1 \%(n=16 / 59)$ in patients who did not develop AKI $(p=0.014)$.

Conclusion. AKI is common in critically ill patients presenting to a tertiary ICU in Durban, SA. AKI is associated with increased mortality and length of stay in the ICU. Strategies to prevent the development or worsening of AKI must be emphasised. These include prevention or at least early treatment of sepsis, adequate fluid resuscitation, aggressive haemodynamic optimisation and avoidance of nephrotoxins. This is especially important in settings where there is limited access to renal replacement therapy (RRT).

Keywords. acute kidney injury; morbidity; mortality; ICU; haemodialysis.
\end{abstract}

South Afr J Crit Care 2020;36(2):80-84. https://doi.org/10.7196/SAJCC.2020.v36i2.426

Contribution of the study. This is one of the first studies to describe the incidence and outcomes of AKI in a general critical care population in a resource-limited setting. The study highlights that AKI is very common in critically ill patients in a resource-limited setting, and is associated with increased mortality and resource utilisation. It also highlights the importance of sepsis as a risk factor for AKI.

Acute kidney injury (AKI) is common in the intensive care unit (ICU) and has a significant impact on morbidity and mortality in critically ill patients. Owing to variations in the definition of AKI, there are conflicting reports on its incidence in the literature. Studies have found that AKI affects $1-25 \%$ of ICU patients, with a reported mortality rate of $15-60 \% .^{[1,2]}$

A large cohort study of ICU patients in Australia showed a significant rise in the incidence of early AKI while the mortality declined. ${ }^{[3]}$ The incidence of AKI was estimated to increase by $2.8 \%$ annually while the crude hospital mortality was significantly higher for patients with AKI compared with those without AKI.

In a multicentre prospective study that enrolled $601 \mathrm{ICU}$ patients, Piccinni et al. ${ }^{[4]}$ found that patients with AKI had a higher crude mortality $(28.8 \%$ v. $8.1 \%)$ and stayed longer in the ICU (median 7 days v. 3 days).

Critically ill patients have multiple aetiological factors that include shock, sepsis and the administration of nephrotoxic agents such as antibiotics and intravenous contrast. 
Theincidence, spectrum and outcomes of AKI in the ICU in South Africa (SA) are unclear. ${ }^{[2,5]}$ The incidence of AKI has been studied in a retrospective study of 666 patients in a trauma unit in South Africa. The study found that AKI had an incidence of $15 \%$ when using the RIFLE classification. ${ }^{[6]}$ Moreover, patients with AKI had an overall mortality of $57 \%$, and $38 \%(n=39)$ of the patients with AKI required renal replacement therapy Another prospective study by Aylward et al..$^{[7]}$ evaluated 849 admissions to the ICU and found that the common reason for admission was trauma (26\%) and that AKI was associated with a higher in-hospital mortality rate of $31.8 \%$ compared with $7.23 \%$ in those without AKI. ${ }^{[7]} \mathrm{A}$ study by Bagshaw et al. ${ }^{[8]}$ found that $42.1 \% \quad(n=14$ 039) of the patients had concomitant AKI and $32.4 \%$ of these patients had sepsis, suggesting that a correlation might exist between AKI and sepsis.

In this study, we sought to describe the burden of AKI among adult SA patients who presented to a tertiary-level ICU. We aimed to determine the impact that AKI has on outcomes such as mortality, stage of AKI severity and length of stay in the ICU.

\section{Methods}

We conducted a retrospective study of 204 patients admitted to the King Edward VIII Hospital ICU from January 2016 to June 2016. This is a multidisciplinary, closed and intensivist-run ICU in a tertiary academic hospital in KwaZulu-Natal Province, SA.

Ethics approval was granted by the University of KwaZulu-Natal Biomedical Research Ethics Committee (ref. no. BE043/18) and the KwaZulu-Natal Department of Health (ref. no. HRKM094/18 KZ_201802_064).

All patients admitted to the ICU were screened for inclusion. The exclusion criteria were children under 18 years of age, patients with previous diagnosis of chronic kidney disease or end-stage renal disease and moribund patients (died within 6 hours of admission to the ICU).AKI was defined and severity graded according to the latest kidney disease improving global outcomes (KDIGO) guidelines using serum creatinine levels. ${ }^{[9]}$

For patients without known prior serum creatinine level, the baseline serum creatinine was estimated using the modification of diet in renal disease (MDRD) equation. ${ }^{[10]}$

Data collection was performed using an Excel spreadsheet and statistical analysis was performed using SPSS Statistics for Windows versions 24.0 and 25.0 (IBM Corp., USA). Descriptive statistics were summarised using frequencies and percentages while continuous data were described using the median and interquartile range (IQR). The $\chi^{2}$ test was used to compare categorical data while the Mann-Whitney U-test was used to compare continuous data. A $p$-value $<0.05$ was considered to be statistically significant.

\section{Results}

We screened 204 patients who were admitted to the ICU and 178 patients were included in the study while 26 were excluded (Fig. 1). More than half of the patients $(56.6 \%$; $n=90 / 178)$ were diagnosed with AKI at the time of admission and 16.3\% $(n=29 / 178)$ developed AKI while in the ICU.

The demographic and outcomes data for the entire cohort are shown in Table 1. More than a quarter of the patients $(32.6 \% ; n=58 / 178)$ had sepsis as their primary diagnosis on admission and $46.1 \%(n=82 / 178)$ had sepsis as one of their ICU diagnoses. Only $19.7 \%$ $(n=35 / 178)$ of the patients were documented to have shock on admission.
AKI and dialysis data are shown in Table 2. Half of the patients $(n=45 / 90)$ who had AKI on admission were classified as KDIGO stage 1, $21.1 \%(n=19 / 90)$ as stage 2 and $28.9 \%$ $(n=26 / 90)$ as stage 3 . The total number of

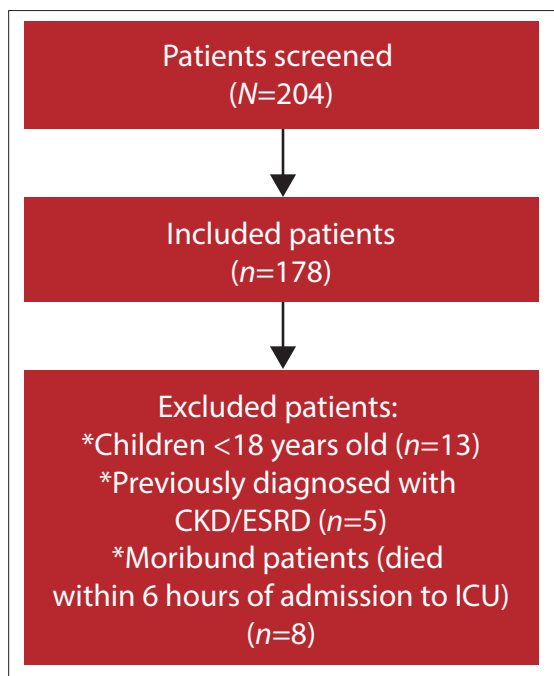

Fig. 1. ROC curve of admission serum albumin and ICU mortality. (CKD = chronic kidney disease; $E S R D=$ end-stage renal disease; $I C U=$ intensive care unit.)

\section{Table 1. Demographic and outcomes data $(N=178)$}

\begin{tabular}{|c|c|}
\hline & $n(\%)^{*}$ \\
\hline Age (years), median (IQR) & $35(26.0-55.0)$ \\
\hline Female gender & $92(51.7)$ \\
\hline \multicolumn{2}{|l|}{ Referring discipline } \\
\hline Medicine & $88(49.4)$ \\
\hline Surgery & $66(37.1)$ \\
\hline O\&G & $24(13.5)$ \\
\hline \multicolumn{2}{|l|}{ Primary admission diagnosis } \\
\hline Sepsis & $58(32.6)$ \\
\hline Trauma & $28(15.7)$ \\
\hline Non-communicable & $92(51.7)$ \\
\hline Sepsis (all) & $82(46.1)$ \\
\hline Shock (documented on admission) & $35(19.7)$ \\
\hline \multicolumn{2}{|l|}{ Comorbidities } \\
\hline Autoimmune disease & $5(2.8)$ \\
\hline Cardiac disease & $20(11.2)$ \\
\hline Chronic kidney disease & $12(6.7)$ \\
\hline Diabetes & $30(16.9)$ \\
\hline HELLP syndrome & $2(1.1)$ \\
\hline \multicolumn{2}{|l|}{ HIV } \\
\hline Positive & $35(19.7)$ \\
\hline Negative & $71(39.9)$ \\
\hline Unknown & $72(40.4)$ \\
\hline Hypertension & $46(25.8)$ \\
\hline \multicolumn{2}{|l|}{ ICU outcome data } \\
\hline ICU mortality & $71(39.9)$ \\
\hline LOS (days), median (IQR) & $4(2.0-8.0)$ \\
\hline LOV (days), median (IQR) & $3(1.0-6.0)$ \\
\hline \multicolumn{2}{|c|}{$\begin{array}{l}\text { IQR = interquartile range; } \mathrm{O} \& \mathrm{G}=\text { obstetrics and gynaecology; HELLP = hemolysis, elevated liver enzymes and low } \\
\text { platelet count; ICU = intensive care unit; } \mathrm{LOS}=\text { length of stay; LOV = length of ventilation. } \\
\text { *Unless otherwise specified. }\end{array}$} \\
\hline
\end{tabular}


Table 2. AKI and dialysis data $(N=178)$

\begin{tabular}{ll}
\hline & $\boldsymbol{n}(\%)^{\star}$ \\
\hline AKI on admission & $90(50.6)$ \\
AKI total & $119(66.9)$ \\
KDIGO stage on admission $(n=90)$ & \\
1 & $45(50.0)$ \\
2 & $19(21.1)$ \\
3 & $26(28.9)$ \\
KDIGO stage total $(n=119)$ & \\
1 & $44(37.0)$ \\
2 & $25(21.0)$ \\
3 & $50(42.0)$ \\
New AKI in ICU & $29(16.3)$ \\
New or worsening AKI in ICU & $51(28.7)$ \\
Dialysed & $29(16.3)$ \\
IHD & $6(20.6)$ \\
SLEDD & $9(31)$ \\
CRRT & $18(62)$ \\
Number of dialysis sessions, $n$ & \\
1 & 6 \\
2 & \\
3 &
\end{tabular}

patients who were classified as KDIGO stage 1,2 and 3 during their stay in the ICU was $24.7 \%(n=44 / 178), 14 \%(n=25 / 178)$ and $28 \%$ ( $n=50 / 178)$, respectively. Finally, only $16.3 \%(n=29 / 178)$ of the patients required dialysis in the ICU and the common indication for dialysis was metabolic acidosis $(82.8 \% ; n=24 / 29)$

Exposure to known or purported nephrotoxins was found in $4.5 \%$ $(n=8 / 178)$ of the patients. Half of these patients $(n=4 / 8)$ required dialysis and these 4 patients represented $13.8 \%$ of the 29 patients who required dialysis.

The results of biochemical investigations on admission are set out in Table 3. Associations between AKI and mortality are shown in Table 4. The mortality rate for patients with AKI on admission was $40.0 \%$ $(n=36 / 90)$ compared with $39.8 \%(n=35 / 88)$ for those without AKI on admission $(p=0.975)$. The mortality rate for all patients with AKI was $46.2 \%(n=55 / 119)$ compared with $27.1 \%(n=16 / 59)$ in patients who did not develop AKI $(p=0.014)$. Patients who developed new or worsening AKI in ICU had a mortality rate of $66.7 \%(n=34 / 51)$ while patients who did not develop AKI or had a stable and improving AKI had a mortality rate of $29.1 \%(n=37 / 127)$.

The median (interquartile range (IQR)) length of stay in the ICU was significantly longer for patients with AKI (5 (3 - 9) days) compared with those without AKI $(3(2-6)$ days; $p<0.001)$. Patients who required dialysis stayed for significantly longer in the ICU (8 (5 - 10) days) compared with those not receiving dialysis $(4(2-6)$ days; $p<0.001)$.

Associations between AKI, demographic data and comorbidity conditions are demonstrated in Table 5.

\section{Discussion}

Our primary aim was to evaluate the incidence and outcomes of AKI as defined by the KDIGO guidelines in our patients. We chose to use the KDIGO guidelines to define AKI as they have been previously validated in critically ill patients. ${ }^{[9]}$

We found the total incidence of AKI to be $66.9 \%$ in our cohort, which is much higher than the $15 \%$ that was reported by Skinner et al. ${ }^{[6]}$ in critically ill trauma patients. A study by Bagshaw et al. ${ }^{[3]}$ found the incidence of AKI to range from $4.6-6.9 \%$ in patients who are in a critical care unit. These differences could be explained by differences in severity of underlying illness, primary diagnosis (in particular sepsis), comorbidities and quality of healthcare systems. In particular, trauma patients are generally young, have few comorbidities and do not have a high incidence of sepsis. The study by Bagshaw et al. ${ }^{[3]}$ presented findings from a developed country where delays to healthcare presentation are less likely and critical care services may be utilised for less severely ill patients.

\section{Table 3. Biochemical data}

\begin{tabular}{|c|c|c|c|c|}
\hline & Entire cohort, median (IQR) & No AKI, median (IQR) & AKI, median (IQR) & $p$-value \\
\hline $\mathrm{Hb}$ & $10.5(8.80-12.10)$ & $10.9(9.10-12.50)$ & $10.4(8.40-12.00)$ & 0.296 \\
\hline $\mathrm{BE}$ & $-4.3(-9.80-1.80)$ & $-1(-5.20-2.80)$ & $-6.5(-11.90-1.30)$ & $<0.001$ \\
\hline Lactate & $2(1.30-3.70)$ & $1.95(1.40-3.20)$ & $2.2(1.30-4.90)$ & 0.354 \\
\hline $\mathrm{BC}$ & $75(55.00-101.00)$ & $61(50.00-78.00)$ & $80(60.00-115.00)$ & $<0.001$ \\
\hline $\mathrm{AC}$ & $113(72.00-190.00)$ & $67(61.00-86.00)$ & $135(98.00-258.00)$ & $<0.001$ \\
\hline $\mathrm{HC}$ & $133(83.00-258.00)$ & $73(62.00-86.00)$ & $198(126.00-392.00)$ & $<0.001$ \\
\hline $\mathrm{AU}$ & $6.5(4.10-13.30)$ & $3.9(2.70-5.30)$ & $10(5.70-17.60)$ & $<0.001$ \\
\hline $\mathrm{HU}$ & $8.4(4.70-16.30)$ & $4.3(3.30-6.70)$ & $12.9(7.70-20.60)$ & $<0.001$ \\
\hline
\end{tabular}

$\mathrm{IQR}=$ interquartile range; $\mathrm{AKI}=$ acute kidney injury; $\mathrm{Hb}=$ haemoglobin; $\mathrm{BE}=$ base excess; $\mathrm{BC}=$ baseline creatinine; $\mathrm{AC}=$ admission creatinine; $\mathrm{HC}=$ highest creatinine; $\mathrm{AU}=$ admission urea; $\mathrm{HU}=$ highest urea. 
Table 4. AKI data and association with mortality $(N=178)$

\begin{tabular}{|c|c|c|c|}
\hline & $\begin{array}{l}\text { Discharged alive } \\
(n=107), n(\%)\end{array}$ & $\begin{array}{l}\text { Died in ICU } \\
(n=71), n(\%)\end{array}$ & $p$-value \\
\hline AKI on admission & $54(50.5)$ & $36(50.7)$ & 0.975 \\
\hline AKI total & $64(59.8)$ & $55(77.5)$ & 0.014 \\
\hline \multicolumn{4}{|l|}{$\begin{array}{l}\text { KDIGO stage on } \\
\text { admission }\end{array}$} \\
\hline 1 & $25(46.3)$ & $20(55.6)$ & 0.385 \\
\hline 2 & $14(25.9)$ & $5(13.9)$ & - \\
\hline 3 & $15(27.8)$ & $11(30.6)$ & - \\
\hline \multicolumn{4}{|l|}{ KDIGO stage total } \\
\hline 1 & $26(40.6)$ & $18(32.7)$ & 0.07 \\
\hline 2 & $17(26.6)$ & $8(14.5)$ & - \\
\hline 3 & $21(32.8)$ & $29(52.7)$ & - \\
\hline \multicolumn{4}{|l|}{$\begin{array}{l}\text { High stage AKI on } \\
\text { admission }\end{array}$} \\
\hline Stage 1 and 2 & $39(72.2)$ & $25(69.4)$ & 0.776 \\
\hline Stage 3 & $15(27.8)$ & $11(30.6)$ & \\
\hline \multicolumn{4}{|c|}{ High stage AKI total } \\
\hline Stage 1 and 2 & $43(67.2)$ & $27(47.3)$ & 0.028 \\
\hline Stage 3 & $21(32.8)$ & $29(52.7)$ & \\
\hline Dialysis in ICU & $18(16.8)$ & $11(15.5)$ & 0.814 \\
\hline New AKI in ICU & $10(9.3)$ & $19(26.8)$ & 0.002 \\
\hline \multicolumn{4}{|c|}{ New or worsening AKI } \\
\hline in ICU & $17(15.9)$ & $34(47.9)$ & $<0.001$ \\
\hline
\end{tabular}

The high incidence of AKI in our study is concerning. Efforts must be intensified to identify patients at risk of disease early and to address modifiable risk factors timeously. Exposure to nephrotoxins is relatively uncommon but is an area of potential intervention. Sepsis is common and is strongly associated with AKI. Emphasis needs to be placed on early diagnosis of infection with timely intervention in terms of starting proper antibiotics and fluid management.

The majority of the patients with AKI had stage 3 disease, indicating worsening AKI during ICU admission. While this may simply reflect a natural evolution of their underlying disease or be a result of the lag in changes in creatinine following initial kidney injury, it may also be due to further insults in ICU. This highlights the importance of adequate resuscitation, maintaining adequate renal perfusion pressures and avoiding nephrotoxins.

The mortality data revealed an association between AKI and mortality. There was no increased mortality in patients with AKI on admission. However, the highest mortality rate was observed in those who developed new or worsening AKI in ICU. This may reflect the patients' response to therapy, with those who respond well to initial ICU therapy having stable or improving creatinine concentrations while the non-responders are more likely to exhibit both worsening of their AKI and increased mortality.

We also hypothesise that increased mortality may be due to complications related to dialysis, which is more likely in patients with worsening AKI. These complications include but are not limited to vascular access, intradialytic hypotension and bleeding from anticoagulant usage. Of note though, the patients who received haemodialysis did not display increased mortality, which suggests that the increased mortality may be due to the severity of the patients' underlying illness. An additional confounder of this data is likely to be selection bias, with clinicians being potentially more likely to offer
Table 5. Association between demographics, disease data and AKI

\begin{tabular}{|c|c|c|c|}
\hline & No AKI & AKI & \\
\hline & $n(\%)^{*}$ & $n(\%)^{*}$ & $p$-value \\
\hline $\begin{array}{l}\text { Age (years), median } \\
\text { (IQR) }\end{array}$ & $\begin{array}{l}30 \\
(23.00-39.00)\end{array}$ & $\begin{array}{l}40 \\
(28.00-59.00)\end{array}$ & $<0.001$ \\
\hline \multicolumn{4}{|l|}{ Gender } \\
\hline Female & $36(61.0)$ & $56(47.1)$ & 0.079 \\
\hline Male & $23(39.0)$ & $63(52.9)$ & \\
\hline \multicolumn{4}{|l|}{ Referring discipline } \\
\hline Medicine & $26(44.1)$ & $62(52.1)$ & 0.323 \\
\hline Surgery & $22(37.3)$ & $44(37.0)$ & \\
\hline O\&G & $11(18.6)$ & $13(10.9)$ & \\
\hline \multicolumn{4}{|l|}{$\begin{array}{l}\text { Primary admission } \\
\text { diagnosis }\end{array}$} \\
\hline Sepsis & $11(18.6)$ & $47(39.5)$ & 0.009 \\
\hline Trauma & $14(23.7)$ & $14(11.8)$ & \\
\hline Non-communicable $^{\dagger}$ & $34(57.6)$ & $58(48.7)$ & \\
\hline Sepsis (all) & $19(32.2)$ & $63(52.9)$ & 0.009 \\
\hline $\begin{array}{l}\text { Shock (documented on } \\
\text { admission) }\end{array}$ & $12(20.3)$ & $23(19.3)$ & 0.873 \\
\hline \multicolumn{4}{|l|}{ Comorbidities } \\
\hline Autoimmune disease & $3(5.1)$ & $2(1.7)$ & 0.334 \\
\hline Cardiac disease & $2(3.4)$ & $18(15.1)$ & 0.020 \\
\hline CKD & $0(0.0)$ & $12(10.1)$ & 0.009 \\
\hline Diabetes & $6(10.2)$ & $24(20.2)$ & 0.093 \\
\hline \multicolumn{4}{|l|}{ HIV } \\
\hline Positive & $13(22.0)$ & $22(18.5)$ & 0.195 \\
\hline Negative & $18(30.5)$ & $53(44.5)$ & \\
\hline Unknown & $28(47.5)$ & $44(37.0)$ & \\
\hline HELLP syndrome & $0(0.0)$ & $2(1.7)$ & 1.000 \\
\hline Hypertension & $12(20.3)$ & $34(28.6)$ & 0.238 \\
\hline Nephrotoxins & $2(3.4)$ & $6(5.0)$ & 1.000 \\
\hline
\end{tabular}

$\mathrm{AKI}=$ acute kidney injury; $\mathrm{O} \& \mathrm{G}=$ obstetrics and gynaecology; $\mathrm{CKD}=$ chronic kidney disease; HELLP = haemolysis, elevated liver enzymes, low platelet count. disease; HELLP = haemolysis,
${ }^{*}$ Unless otherwise specified.

${ }^{*}$ *nless otherwise specified. ${ }^{\dagger} \mathrm{N}$ Non-communicable disease: non-infectious, non-traumatic diseases that may include
but are not limited to non-infectious complications of diabetes mellitus, e.g. diabetic ketoacidosis, myocardial infarction, cerebrovascular accident, non-infectious exacerbations of chronic pulmonary disease e.g. asthma, drug overdose.

dialysis to patients with a greater likelihood of survival as opposed to those with a poor prognosis. These hypotheses should be explored in future studies.

The median ICU length of stay was longer in patients with AKI compared with those without AKI. However, patients receiving dialysis had a significantly longer length of stay in the ICU compared with those not receiving dialysis. The greater length of stay may reflect increased severity of illness. However, given the limited access to dialysis facilities outside the ICU, this may indicate a need to keep patients in the ICU to ensure continuous haemodialysis. These findings emphasise the burden of AKI and a need for dialysis in critical care services. Moreover, this also highlights a need to ensure availability of dialysis facilities outside the ICU to prevent the need for ICU in some instances and delayed step-down from ICU.

The relatively low rate of dialysis in our study of $16.3 \%(n=29 / 178)$ or $24.4 \%$ of all patients with AKI is noteworthy and contrasts with a previously reported rate of $36.5 \% .^{[11]}$ This may be because of a preponderance of low-stage AKI that did not require dialysis. Other possible explanations for the low rate of dialysis are the triage decisions of the treating team not to offer dialysis to patients with a poor prognosis and possible limitations in the availability of dialysis. 


\section{Study limitations}

This is a single-centre retrospective study and may have limited generalisability. However, the study was conducted in a referral ICU at the second most populous province in SA, so there is a possibility that the findings may reflect more general practices. We had limited premorbid data for some patients, which resulted in difficulties in estimating the baseline creatinine levels. We used the MDRD equation to estimate the baseline creatinine, but this estimates a high normal glomerular filtration rate and could potentially be inaccurate in those with both higher and lower baseline glomerular filtration rates. In general, the MDRD equation tends to over-diagnose AKI. ${ }^{[12]}$ Renal ultrasound was not routinely performed and the lack of ultrasound data to determine the size of kidneys before inclusion into the study may have led to some patients with chronic kidney disease being incorrectly diagnosed with AKI. Timing and volume of fluid administration prior to serum samples being drawn was not evaluated, which could potentially have altered the biochemical results. Indications for dialysis are not protocolised in the ICU and, given the retrospective nature of the study, the indications for dialysis described in this article were presumptive.

\section{Conclusion}

AKI is common in critically ill patients presenting to a tertiary ICU in SA. AKI is associated with increased mortality and length of stay. The need for dialysis in ICU is associated with prolonged length of stay and represents a significant healthcare burden.

Declaration. None.

Acknowledgements. None.
Author contributions. MAK: study design, data collection, data interpretation, drafted study manuscript. KdV and DS: study design, data analysis, data interpretation, reviewed manuscript. All authors approved the manuscript for publication.

Funding. None.

Conflicts of interest. None.

1. Ostermann M, Chang RW. Acute kidney injury in the intensive care unit according to RIFLE. Crit Care Med 2007;35(8):1837-1843. https://doi.org/10.1097/01.ccm.0000277041.13090.0a

2. Skinner DL, Kong VY, de Vasconcellos K, et al. Acute kidney injury on presentation to a major trauma service is associated with poor outcomes. J Surg Res 2018;232:376-382. https://doi. org/10.1016/j.jss.2018.06.069

3. Bagshaw SM, George C, Bellomo R. Changes in the incidence and outcome for early acute kidney injury in a cohort of Australian intensive care units. Critical Care 2007;11(3):R68. https://doi.
inger injury in a cohort of Austa
$\mathrm{org} / 10.1186 \% 2 \mathrm{Fcc} 5949$

4. Piccinni P, Cruz D, Gramaticopolo S, et al. Prospective multicentre study on epidemiology of acute kidney injury in the ICU: A critical care nephrology Italian collaborative effort (NEFROINT). Minerva Anestesiologica 2011;77(11):1072.

5. Bowley DM, Buchan C, Khulu L, Boffard KD. Acute renal failure after punishment beatings. Royal Society Med 2002;95(6):300-301. https://doi.org/10.1258\%2Fjrsm.95.6.300

6. Skinner D, Hardcastle T, Rodseth R, Muckart D. The incidence and outcomes of acute kidney injury among patients admitted to a level I trauma unit. Injury 2014;45(1):259-264. https://doi. org/10.1016/j.injury.2013.07.013

7. Aylward RE, van der Merwe E, Pazi S, et al. Risk factors and outcomes of acute kidney injury in South African critically ill adults: A prospective cohort study. BMC Nephrol 2019;20(1):460. https://doi.org/10.1186/s12882-019-1620-7

8. Bagshaw SM, George C, Bellomo R. Early acute kidney injury and sepsis: A multicentre evaluation. Critical Care 2008;12(2):R47. https://doi.org/10.1186/cc6863

9. Kideny international supplements KDIGO clinical practice guideline for acute kidney injury. http://www.kidney-international.org. March 2012;2(1).

10. Khwaja A. KDIGO clinical practice guidelines for acute kidney injury. Nephron Clinical Practice 2012;120(4):c179-c184. https://doi.org/10.1159/000339789

11. de Abreu KLS, Júnior GBS, Barreto AG, et al. Acute kidney injury after trauma: Prevalence, clinical characteristics and RIFLE classification. Indian J Crit Care Med 2010;14(3):121. https:// doi.org/10.4103\%2F0972-5229.74170

12. Pickering JW, Endre ZH. Back-calculating baseline creatinine with MDRD misclassifies acute kidney injury in the intensive care unit. Clin J American Society Nephrol 2010;5(7):1165-1173. https://doi.org/10.2215/cjn.08531109

Accepted 27 May 2020. 